\section{STAP using Knowledge-Aided Covariance Estimation and the FRACTA Algorithm}

\author{
SHANNON D. BLUNT, Member, IEEE \\ University of Kansas
}

KARL GERLACH, Fellow, IEEE

U.S. Naval Research Laboratory

MURALIDHAR RANGASWAMY, Fellow, IEEE

U.S. Air Force Research Laboratory

In the airborne space-time adaptive processing (STAP) setting, a priori information via knowledge-aided covariance estimation

(KACE) is employed in order to reduce the required sample support for application to heterogeneous clutter scenarios. The enhanced FRACTA (FRACTA.E) algorithm with KACE as well as Doppler-sensitive adaptive coherence estimation (DS-ACE) is applied to the KASSPER I \& II data sets where it is shown via simulation that near-clairvoyant detection performance is maintained with as little as $1 / 3$ of the normally required number of training data samples. The KASSPER I \& II data sets are simulated high-fidelity heterogeneous clutter scenarios which possess several groups of dense targets. KACE provides a priori information about the clutter covariance matrix by exploiting approximately known operating parameters about the radar platform such as pulse repetition frequency (PRF), crab angle, and platform velocity. In addition, the DS-ACE detector is presented which provides greater robustness for low sample support by mitigating false alarms from undernulled clutter near the clutter ridge while maintaining sufficient sensitivity away from the clutter ridge to enable effective target detection performance.

Manuscript received December 18, 2004; revised June 10, 2005; released for publication January 17, 2006.

IEEE Log No. T-AES/42/3/879010.

Refereeing of this contribution handled by W. L. Melvin.

This work was supported by the United States Office of Naval Research ONR 31 and Dr. Joseph Guerci of DARPA.

Authors' addresses: S. D. Blunt, University of Kansas, 2335 Irving Hill Rd., Lawrence, KS 66045; K. Gerlach, U.S. Naval Research Laboratory, 4555 Overlook Ave. S.W., Washington, D.C. 20375; M. Rangaswamy, U.S. Air Force Research Laboratory/SNHE, 80 Scott Dr., Hanscom AFB, MA 01731.

0018-9251/06/\$17.00 (c) 2006 IEEE

$\begin{array}{ll}\text { ACE } & \text { Adaptive coherence estimate } \\ \text { AMF } & \text { Adaptive matched filter } \\ \text { APR } & \text { Adaptive power residue } \\ \text { CA-CFAR } & \text { Cell-averaging constant false alarm rate } \\ \text { CBP } & \text { Concurrent block processing } \\ \text { CPI } & \text { Coherent processing interval } \\ \text { CSR } & \text { Censoring stopping rule (FRACTA } \\ & \text { enhancement) } \\ \text { CTD } & \text { Censored training data } \\ \text { DS-ACE } & \text { Doppler-sensitive ACE } \\ \text { CUT } & \text { Cell-under-test } \\ \text { FML } & \text { Fast maximum likelihood } \\ \text { FRACTA } & \text { FML, RC, APR, CBP, TWM, and ACE } \\ \text { FRC } & \text { Fast RC (FRACTA enhancement) } \\ \text { GC } & \text { Global censoring (FRACTA } \\ & \text { enhancement) } \\ \text { GCM } & \text { General clutter model } \\ \text { ITD } & \text { Initial training data } \\ \text { KACE } & \text { Knowledge-aided covariance estimation } \\ \text { KASSPER } & \text { Knowledge-aided sensor signal } \\ & \text { processing and expert reasoning } \\ \text { LSMI } & \text { Loaded sample matrix inverse } \\ \text { RC } & \text { Reiterative censoring } \\ \text { SWP } & \text { Sliding window processing } \\ \text { TWM } & \text { Two-weight method } \\ \text { UTD } & \text { Uncensored training data. } \\ & \end{array}$

\section{INTRODUCTION}

A fundamental issue in space-time adaptive processing (STAP) for the airborne/space-based radar scenario is the accurate estimation of the clutter covariance matrix. In the presence of severely heterogeneous interference, it is desired to employ low sample support for covariance matrix estimation. For low (or small) enough sample support it can be assumed that the interference in the training data is locally stationary (representative) thereby inhibiting smearing of the interference covariance estimate. However, a certain nominal amount of data is required to estimate effectively the interference covariance which, in the case of low sample support, must be supplemented by some other means. In this paper, we investigate the use of a priori knowledge of the clutter covariance matrix to maintain a prescribed performance at low sample support.

Under the condition of low sample support, we examine the performance of the enhanced FRACTA (FRACTA.E) algorithm [1-4] when supplemented with knowledge-aided covariance estimation (KACE) [5-7] and the Doppler-sensitive adaptive coherence estimate (DS-ACE) detector [8]. FRACTA.E is a "meta-algorithm" comprised of simpler algorithms and metrics, some of which can be shown to possess optimality properties. 
For the airborne/space-based radar setting, the FRACTA.E algorithm has been demonstrated [3, 4] to be proficient at excising outliers that closely resemble targets (i.e., that match the desired steering vector) from the training data thereby enabling the effective detection of densely clustered targets in the presence of heterogeneous interference in an efficient manner. In this paper, the FRACTA.E algorithm is supplemented with KACE and DS-ACE and applied to the knowledge-aided sensor signal processing and expert reasoning (KASSPER) I \& II $[9,10]$ datasets $^{1}$ in order to ascertain the benefit of employing estimated prior knowledge when low sample support is required. The KASSPER datasets were generated by a high-fidelity clutter model developed by the Defense Advanced Research Projects Agency (DARPA) and the Air Force Research Laboratory (AFRL) and possess several groups of densely spaced moving ground targets which cause severe performance degradation for standard adaptive matched filter (AMF) processors. Previously, the original FRACTA algorithm was shown [2] to perform well for environments with dense targets, heterogeneous clutter, and jamming for three databases: for the RLSTAP [11] and KASSPER I [9] simulated databases and the multi-channel airborne radar measurements (MCARM) [12] measured database.

The airborne radar space-time adaptive processing (STAP) configuration is comprised of a radar antenna system that consists of an $\mathrm{N}$-element uniform linear array that provides $N$ RF input signals or channels. Time delayed samples of these $N$ inputs are to be combined via AMF linear weighting [13]. The time delayed samples correspond to $M$ identical pulses with pulse repetition interval (PRI) equal to $T$ which constitute the coherent processing interval (CPI). Hence, there is a length $M N$ space-time sample vector corresponding to each individual range cell.

The solution to the AMF problem results in a maximization of the output signal-to-noise ratio (SNR). The maximum output SNR is attained when the covariance matrix is perfectly known thereby yielding clairvoyant filter weights. Due to the lack of knowledge of the external environment, adaptive techniques require a certain amount of data to estimate the $M N \times M N$ covariance matrix effectively. The amount of data (the number of statistically independent and identically distributed (IID) samples per input sensor) required so that the performance of the adaptive processor is within $3 \mathrm{~dB}$ of the optimum is called the convergence measure of effectiveness (MOE) of the processor. It was shown

\footnotetext{
${ }^{1}$ Available by request from AFRL/SNRT, 26 Electronic Pky., Rome, NY 13441-4514.
}

in [14] that for the standard sample matrix inverse (SMI) of a full rank $M N \times M N$ matrix the MOE is $K=2 M N$ samples. Further reducing the convergence $\mathrm{MOE}$ is important since the characteristics of the external interference change rapidly with space and time in many environments which has led to the development of techniques such as loaded sample matrix inverse (LSMI) [15], principal components inverse [16, 17], the multistage Wiener filter $[18,19]$, fast maximum likelihood (FML) [20], and reduced dimensionality STAP [21]. The convergence MOE for these techniques is approximately $2 J$ where $J<M N$ is the number of dominant eigenvalues of the $M N \times M N$ covariance matrix.

Typically for adaptive radar applications, the sample covariance matrix is estimated using training data from range cells close to the range cell under test (CUT) under the assumption that the second-order statistics of the surrounding range cells are relatively homogeneous with the CUT. For the standard sliding window processor (SWP) method, the covariance matrix is recomputed for each CUT with a number of guard cells (usually between 3 and 7) on each side of the CUT excluded from the training data. However, the presence of outliers in the training data can skew the covariance matrix estimate such that a true target in the CUT is suppressed. It has been shown [1-3] that the FRACTA algorithm, through the use of concurrent block processing (CBP), reiterative adaptive power residue (APR) censoring, and adaptive coherence estimate (ACE) detection, is robust to target-like outliers in the training data and performs effectively even in the presence of dense groups of targets. Furthermore, the censoring stage of the FRACTA algorithm has since been enhanced [4] (resulting in FRACTA.E) such that the numerical efficiency surpasses the standard SWP methodology.

Besides outliers in the training data, another obstacle to realizing effective STAP performance is nonstationarity of the second-order statistics of range cells near the CUT, which gives rise to nonrepresentative training data. Employing nonrepresentative training data for the estimation of the interference covariance matrix results in reduced probability of detection and increased probability of false alarm due to undernulled interference [22]. On the other hand, the reduction in the sample support in order to maintain homogeneity can cause the processor to be deficient of the samples necessary to attain the convergence MOE.

To combat the effects of heterogeneous nonstationary interference, this paper revisits the concept of KACE [5, 6] which is based upon the a priori "bald Earth" radar clutter scenario established in [23]. For certain known (or estimated) radar operating parameters, KACE generates an estimate of the clutter covariance matrix which can be used to supplement the covariance matrix estimate obtained 
from the training data in order to substantially reduce the necessary sample support in heterogeneous environments. To enable further robustness at low sample support, this paper also develops the DS-ACE detector which was briefly introduced in [8]. The ACE detector [24] yields a test statistic for each individual range/Doppler cell and is known to be quite effective at discriminating between targets and false alarms that result from space-time filter sidelobes. The DS-ACE detector computes jointly the ACE thresholds for different Doppler bands according to their individual proximity to the clutter ridge. As a result, undernulled clutter that occurs due to insufficient sample support does not cause undue false alarms while sensitivity is still maintained for the detection of targets off of the clutter ridge peak.

Other STAP approaches have been proposed which employ a priori knowledge in one way or another. In [25] Melvin et al. discussed the use of prior knowledge from databases such as digital terrain elevation data (DTED), synthetic aperture radar, hyperspectral imagery, etc. to enhance the overall STAP architecture. Prewhitening of received radar data using a priori knowledge was discussed in [26]. Additional work on knowledge-aided STAP can be found in, for example, [27]-[30].

The remainder of the paper is organized as follows. Section II presents a brief overview of the FRACTA.E algorithm. Section III discusses KACE and its application to FRACTA.E. In Section IV the DS-ACE detector is introduced. Finally, Section V presents simulation results from applying the FRACTA.E-KACE algorithm to the KASSPER I \& II datasets.

\section{THE FRACTA ALGORITHM}

FRACTA is an acronym for the systematic combination of the following algorithms or methods: FML algorithm, reiterative censoring (RC), the adaptive power residue (APR metric), concurrent block processing (CBP), two-weight method (TWM) computation, and the adaptive coherence estimate (ACE) metric. FRACTA is therefore a "meta-algorithm" that is comprised of several algorithms/detectors that perform the individual functions of censoring outliers from the data, covariance matrix estimation, target detection, etc. Although no optimality claims for FRACTA are made here or elsewhere, it is found to yield effective performance in heterogeneous clutter scenarios.

Given the length- $M N$ space-time steering vector $\mathbf{s}$ (which is equal to the Kronecker product of the $M$-length desired temporal steering vector and the $N$-length desired spatial steering vector [23]), it is well known [13] that the conjugate weighting that maximizes the signal-to-interference power ratio of the length- $M N$ snapshot (or data vector) $\mathbf{z}$ associated with the CUT is given by

$$
\mathbf{w}=\mathbf{R}^{-1} \mathbf{s}
$$

where $\mathbf{R}$ is the true $M N \times M N$ interference covariance matrix for the CUT.

In practice, $\mathbf{R}$ is not known and must be estimated from the available data (typically from the length- $M N$ sample vectors associated with the range cells surrounding the CUT exclusive of guard cells). The FML algorithm [20] is a "reduced-rank" approach based upon a maximum likelihood formulation and is used to estimate the unknown covariance matrix from the $K$ surrounding data vectors $(K \ll 2 M N$ snapshots that are required for SMI [14]). The FML covariance matrix is assumed to be composed of a known diagonal matrix (without loss of generality assumed to be identity after normalization by the internal system noise power) plus an unknown positive semi-definite Hermitian matrix.

Some of the $K$ training data vectors $\mathbf{z}_{1}, \mathbf{z}_{2}, \ldots, \mathbf{z}_{K}$, used by FML to estimate the interference covariance matrix may contain outliers that coalign with the space-time steering vector $\mathbf{s}$. Censoring of the training data is therefore required so that potential targets are not suppressed. The censoring of outliers for a block of training data is accomplished by first computing the APRs for the $K$ data vectors within the data block as

$$
\text { APR: } \quad\left\{\left|\mathbf{s}^{H} \tilde{\mathbf{R}}^{-1} \mathbf{z}_{k}\right|^{2}\right\}, \quad k=1,2, \ldots, K
$$

in which $\tilde{\mathbf{R}}$ is the covariance matrix estimated by FML from the set of $K$ data vectors and $(\cdot)^{H}$ is the complex conjugate transpose (or Hermitian) operation. Applied in this way, the APR metric is a relative measure of how closely each snapshot matches the desired steering vector thereby providing an indication as to the potential presence of a target. The snapshot with the largest APR is removed (i.e., censored) from the set of training data, FML is used to estimate the covariance matrix from the remaining $K-1$ data vectors, and then the process is repeated reiteratively (hence RC). This continues until a predetermined number of snapshots have been censored; either a maximum allowable number of censored snapshots or when some stopping criteria is reached. The stopping criteria is met when a probe vector which is appended to the training data set is identified as the snapshot with the largest APR. The probe vector takes the form

$$
\text { probe: } \alpha_{p} \mathbf{s}
$$

where $\alpha_{p}$ is a predetermined magnitude that is set such that the probe vector is nominally detectable (10-15 dB above the noise floor after integration).

$\mathrm{RC}$ is performed globally in range whereby, for a given space-time steering vector, all range cells are included in the training data set. RC is then performed for each individual Doppler frequency. Global censoring (GC), as opposed to local censoring 
on smaller blocks of the training data, is especially useful in areas where dense clusters of targets are present such that the covariance matrix estimate would otherwise be skewed if the block of data were not sufficiently large. The presence of many targets in an insufficiently large data block can result in low APR values for the targets and therefore hinder proper censoring of targets.

As noted previously, the SWP approach exacts a high computational cost due to repeated matrix inversions. The use of CBP greatly alleviates much of the computational burden experienced by SWP by eliminating the guard cells and designating a block of $K_{\mathrm{CUT}}$ data vectors within the set of $K$ training data vectors as pertaining to a set of CUTs. The consolidation of a set of CUTs into a single training data block effectively reduces by a factor of $K_{\text {CUT }}$ the number of times that covariance matrix estimation/inversion and censoring need to be undertaken thereby substantially reducing computation. Also, the use of RC eliminates the need for guard cells by adaptively removing target-like snapshots from the training data. By a process of $\mathrm{RC}$, each data vector in the set of initial training data (ITD), which consists of all $K$ training data vectors, is assigned to either the set of $K_{\mathrm{C}}$ censored training data (CTD) which appear to be target-like snapshots or the set of $K_{\mathrm{U}}$ uncensored training data (UTD). Thus $\mathrm{ITD}=\mathrm{UTD} \cup \mathrm{CTD}$.

To enable the censored potential targets to stand out further from the quiescent noise level, two adaptive weight vectors are computed; one each for the CTD and the UTD. The CTD weight vector $\mathbf{w}_{\mathrm{C}}$ is computed using the UTD covariance matrix estimate $\tilde{\mathbf{R}}_{\mathrm{U}}$ as

$$
\mathbf{w}_{\mathrm{C}}=\tilde{\mathbf{R}}_{\mathrm{U}}^{-1} \mathbf{s}
$$

because it is assumed that all snapshots that resemble targets have been removed. The UTD weight vector $\mathbf{w}_{\mathrm{U}}$ is computed using the ITD covariance matrix $\tilde{\mathbf{R}}_{\mathrm{I}}$ estimated from the complete set of $K$ training data vectors as

$$
\mathbf{w}_{\mathrm{U}}=\tilde{\mathbf{R}}_{\mathrm{I}}^{-1} \mathbf{s}
$$

since the UTD is assumed to contain no snapshots with targets and the ITD has more samples than the UTD thus providing a more accurate estimate of the covariance matrix. The weight vectors $\mathbf{w}_{C}$ and $\mathbf{w}_{U}$ are then applied to the respective censored and uncensored snapshots that constitute the set of CUTs. This procedure is denoted as the TWM. It was shown in [1] that TWM yields better performance than the standard one-weight method.

The use of TWM results in targets standing out dramatically from the suppressed noise and interference thus greatly improving the detectability of small target returns. Care must be taken, however, because censored cells that do not contain targets

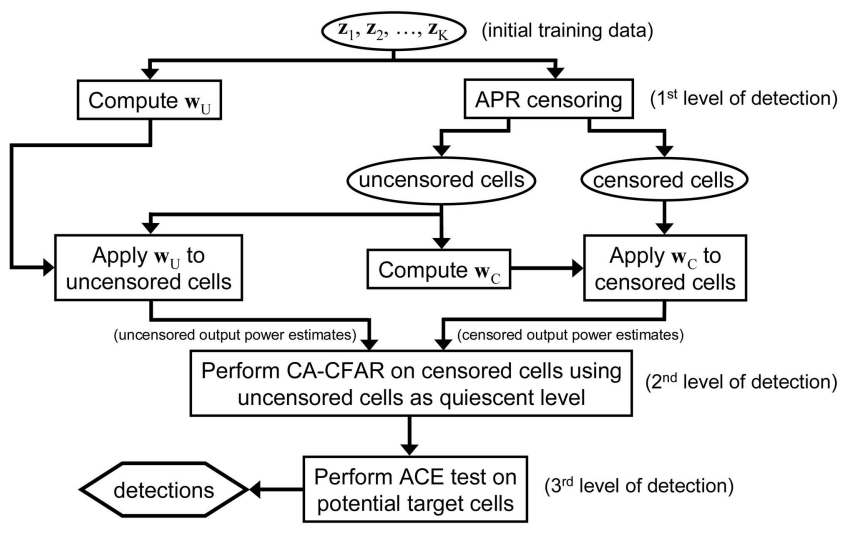

Fig. 1. General FRACTA operation.

will stand out somewhat as well. For this reason, in addition to the usual cell-averaging constant false alarm rate (CA-CFAR) detector, the ACE metric is also employed as a measure of target detection. The ACE metric for range index $k$ is defined as [24]

$$
\operatorname{ACE}(k)=\frac{\left|\mathbf{s}^{H} \tilde{\mathbf{R}}_{k}^{-1} \mathbf{z}_{k}\right|^{2}}{\left(\mathbf{s}^{H} \tilde{\mathbf{R}}_{k}^{-1} \mathbf{s}\right)\left(\mathbf{z}_{k}^{H} \tilde{\mathbf{R}}_{k}^{-1} \mathbf{z}_{k}\right)}
$$

where $\tilde{\mathbf{R}}_{k}$ is the respective covariance matrix (i.e., $\tilde{\mathbf{R}}_{\mathrm{I}}$ or $\tilde{\mathbf{R}}_{\mathrm{U}}$ ) for the $k$ th range index and Doppler of interest. The ACE screens out potential detections that are not coaligned with the desired steering vector $\mathbf{s}$, such as undernulled clutter or targets in the antenna/Doppler filter sidelobes.

A functional block diagram of the FRACTA algorithm as a whole is shown in Fig. 1. The complete detection mechanism used for the FRACTA algorithm consists of three detection stages: censoring, CA-CFAR, and ACE. APR censoring is applied to the set of ITD $\left\{\mathbf{z}_{1}, \mathbf{z}_{2}, \ldots, \mathbf{z}_{K}\right\}$ such that the ITD is divided into a set of UTD and a set of CTD. The UTD is used to compute the adaptive weight vector $\mathbf{w}_{C}$ which is applied to the CTD to generate the censored output power residues. The ITD is used to compute the adaptive weight vector $\mathbf{w}_{\mathrm{U}}$ which is applied to the UTD to generate the uncensored output power residues. The censored output power residues are subjected to a local CA-CFAR detector which uses the uncensored output power residues in the proximity of the CUT to estimate the quiescent level. The cells passing the CFAR detector are then tested with an ACE detector that compares the ACE of the respective candidate cells with some predetermined threshold. Cells that pass the ACE detector are declared as detections.

In [4], enhancements to the FRACTA algorithm were presented which greatly increase its numerical efficiency as well as provide some improvement in performance. Specifically, these enhancements pertain to censoring which is the most computational intensive portion of the FRACTA algorithm as well as 
being the first level of detection. The enhancements consists of GC, fast reiterative censoring (FRC), and censoring stopping rule (CSR). GC involves using a large block (if not all) of the data vector samples to perform censoring for each individual Doppler under the assumption that cells containing targets constitute a relatively small portion of the total number of cells. Hence, target cells tend to be nulled the least and therefore will most likely possess the largest APR values. Fast RC involves the replacement of the FML algorithm with the well-known LSMI for which $\mathrm{RC}$ of the data sample vectors from the covariance matrix estimate is then accomplished by means of Woodbury's identity [31], thereby eliminating the need for repeated matrix inversions. Finally, the CSR appends a vector to the training data that takes the form of the desired steering vector scaled to be nominally detectable (say 10-15 dB). When the probe is censored, it is assumed that all detectable target cells have been censored for the particular Doppler, thus censoring is halted. Also, potential false alarms are reduced due to fewer overall censored cells. The FRACTA algorithm, with these enhancements, is denoted as the FRACTA.E algorithm. Additional information regarding the FRACTA.E algorithm can be found in [32].

\section{KNOWLEDGE-AIDED COVARIANCE ESTIMATION}

KACE employs partial approximate knowledge of the clutter covariance matrix based upon the simplified general clutter model (GCM) [5, 15]. The spatial input channels are assumed to be highly matched (or calibrated). Prior knowledge is assumed for the number of antenna elements and pulses in the CPI, the radar $\beta$ parameter [23] (the number of half-wavelengths traversed by the platform between successive pulses which measures the slope of the clutter ridge in azimuth-Doppler), the crab angle, the clutter power, the model for the intrinsic clutter motion (i.e., Gaussian [33], Billingsley [34], etc.) and its associated parameters, the element-spacing to wavelength ratio, an antenna transmit pattern (approximate), and the look direction azimuth and depression angles. All of these are either system design parameters or are readily measurable (but not necessarily accurate). In general, at a given nominal range from the radar, the KACE covariance matrix takes the form [6]

$$
\tilde{\mathbf{R}}_{\mathrm{KACE}}=\sum_{\ell=1}^{N_{c}} \xi_{\ell}\left(\boldsymbol{\Gamma}_{\ell} \odot \mathbf{b}_{\ell} \mathbf{b}_{\ell}^{H}\right) \otimes\left(\mathbf{a}_{\ell} \mathbf{a}_{\ell}^{H}\right)
$$

where $N_{c}$ is the number of independent clutter patches evenly distributed in azimuth, $\xi_{\ell}, \boldsymbol{\Gamma}_{\ell}, \mathbf{b}_{\ell}$, and $\mathbf{a}_{\ell}$ are the power, intrinsic clutter covariance matrix (ICM), $M \times 1$ temporal steering vector, and $N \times 1$ spatial steering vector, respectively, of the $\ell$ th clutter patch which are functions of the a priori parameters listed previously. The operators $\odot$ and $\otimes$ are the Hadamard and Kronecker matrix product operators, respectively. The $N$-length temporal and $M$-length spatial steering vectors are defined as

$$
\begin{array}{r}
\mathbf{a}_{\ell}(n)=\exp \left(j(n-1) 2 \pi \frac{d}{\lambda_{0}} \cos \theta_{\ell} \sin \phi_{\ell}\right), \\
\ell=1,2, \ldots, N_{c}, \quad n=1,2, \ldots, N
\end{array}
$$

and

$$
\begin{gathered}
\mathbf{b}_{\ell}(m)=\exp \left(j(m-1) 2 \pi \frac{d}{\lambda_{0}} \beta \cos \theta_{\ell} \sin \left(\phi_{\ell}+\phi_{a}\right)\right), \\
\ell=1,2, \ldots, N_{c}, \quad m=1,2, \ldots, M
\end{gathered}
$$

where $d$ is the distance between adjacent antenna elements, $\lambda_{0}$ is the wavelength of the center frequency of the transmitted waveform, $\left(\phi_{\ell}, \theta_{\ell}\right)$ is the true azimuth and depression angles of the $\ell$ th clutter patch measured from the reference of the antenna platform, $\phi_{a}$ is the misalignment angle (or crab angle), and $\beta=$ $2 v T / d$ in which $v$ is the platform velocity. Following the development in $[5,6]$, the ICM is modeled by a constant $M \times M$ matrix, $\Gamma$, using the Gaussian clutter spectral model [33] where

$$
\Gamma_{q, r}=\rho^{(q-r)^{2}}, \quad q, r=1,2, \ldots, M
$$

where $\rho$ is the clutter correlation parameter and is necessarily close to 1 . Finally, known array channel mismatch effects as a function of $\phi_{\ell}$ and $\theta_{\ell}$ can be included in $\mathbf{b}_{\ell}(m)$, albeit these effects are not included in the present formulation of $\tilde{\mathbf{R}}_{\mathrm{KACE}}$.

For the bald Earth model the clutter patch powers are assumed to be uniform in azimuth and are set according to the average measured clutter power over range. Hence, $\tilde{\mathbf{R}}_{\mathrm{KACE}}$ is scaled such that each of its diagonal elements is the average of the diagonal elements of the data-estimated covariance matrix $\tilde{\mathbf{R}}$. Of course, superior performance could be expected if the individual patch powers for each particular range cell were available via some a priori knowledge database. However, this would necessitate the calculation of $\tilde{\mathbf{R}}_{\mathrm{KACE}}$ for each individual range cell which is quite computationally burdensome.

$\mathrm{KACE}$ is employed in the computation of the AMF weight vectors as [5]

$$
\hat{\mathbf{w}}=\left(\tilde{\mathbf{R}}+\tilde{\mathbf{R}}_{\mathrm{KACE}}\right)^{-1} \mathbf{s}
$$

such that the effective covariance matrix is comprised of both clutter covariance information measured directly from the environment in the form of the data vector samples and estimated a priori information via $\mathrm{KACE}$. We utilize KACE to supplement the 
FRACTA.E algorithm under the condition of low sample support due to heterogeneous interference. Thus $\tilde{\mathbf{R}}_{\mathrm{KACE}}$ acts as a colored loading term similar to that used in [26] and [35] which provides an approximation to the information contained in the data-estimated clutter covariance matrix $\tilde{\mathbf{R}}$ thereby enabling sample support lower than the usual $2 J$ samples required by standard rank reduction techniques [15-20].

The a priori clutter covariance matrix $\tilde{\mathbf{R}}_{\mathrm{KACE}}$ resulting from the bald Earth approximation and Gaussian clutter spectral model for the ICM is by definition slightly mismatched to the true clutter covariance matrix R. In addition, radar platform parameters such as crab angle and platform velocity are estimates and thus may contain errors. However, as discussed in [5], there are still important features of the true clutter covariance matrix that are incorporated into $\tilde{\mathbf{R}}_{\mathrm{KACE}}$. Furthermore, the part of $\mathbf{R}$ that is not known or properly incorporated into $\tilde{\mathbf{R}}_{\mathrm{KACE}}$ is contained partially in the data-estimated covariance matrix $\tilde{\mathbf{R}}$. The use of $\tilde{\mathbf{R}}_{\mathrm{KACE}}$ thus provides a means of (at least approximately) estimating the true covariance matrix $\mathbf{R}$ when too few samples are available due to clutter heterogeneity.

\section{DOPPLER-SENSITIVE ACE DETECTOR}

The ACE detector, which is the last detection stage for the FRACTA.E algorithm, has been found to be effective at discriminating true targets from large returns associated with space-time sidelobes, thereby minimizing false alarms $[2,24]$. In general, the ACE values close to the clutter ridge in Doppler tend to be larger than those farther away due to the possible presence of undernulled clutter. As the sample support for the AMF weight vector estimation decreases, the amount of undernulled clutter subsequently increases. Hence, it would be prudent to employ an ACE threshold that is Doppler dependent and increases according to its proximity to the clutter ridge in order to improve the robustness of FRACTA.E to undernulled clutter.

It is known that for side-mounted antenna arrays and small crab angle, the Doppler frequency of the clutter ridge for the spatial direction of interest is approximately [23]

$$
f_{c}\left(\theta_{\text {look }}, \phi_{\text {look }}\right)=\frac{2 v}{\lambda_{0}} \cos \theta_{\text {look }} \sin \phi_{\text {look }}
$$

where $\theta_{\text {look }}$ and $\phi_{\text {look }}$ are the depression and azimuth angles for the spatial look direction, respectively. At a given spatial look direction, when the clutter ridge Doppler frequency is higher than PRF/2 (such as may occur with low PRF radars), the clutter ridge is aliased to the Doppler frequency

$$
f_{c, \text { alias }}=\left[\left(f_{c}+\frac{\mathrm{PRF}}{2}\right) \operatorname{modPRF}\right]-\frac{\mathrm{PRF}}{2}
$$

where $x \bmod y$ represents the " $x$ modulo $y$ " operation.

For each individual Doppler bin, the ACE threshold should be set according to its proximity to the clutter ridge (taking into account the potential wrap-around in Doppler frequency) and the desired false alarm probability. Hence, a large ACE threshold should be set for Dopplers close to the clutter ridge and the Dopplers further from the clutter ridge are then allowed to have a relatively low ACE threshold thereby maintaining the sensitivity to detect targets. This methodology is denoted as the DS-ACE detector.

The DS-ACE methodology can be implemented in a data-dependent manner by taking a CFAR-like approach (i.e., setting the respective ACE thresholds according to the surrounding quiescent ACE levels). It is assumed that local snapshots (in terms of range) that may potentially contain a target have been censored such that the set of $K$ ITD snapshots has been separated into a set of $K_{c}$ CTD snapshots and a set of $K_{u}$ UTD snapshots (where $K=K_{c}+K_{u}$ ). As with (4) and (5), two covariance matrix estimates are computed; $\tilde{\mathbf{R}}_{\mathrm{U}}$, the UTD covariance matrix estimate comprised of only the uncensored local snapshots, and $\tilde{\mathbf{R}}_{\text {I }}$, the ITD covariance matrix estimate composed of both censored and uncensored local snapshots. The UTD covariance matrix estimate $\tilde{\mathbf{R}}_{\mathrm{U}}$ is employed in (6) to compute the ACE values for the block of $k_{c}=1,2, \ldots, K_{c}$ local censored snapshots $\mathbf{z}_{\mathrm{CTD}, k_{c}}$ for each of the $m=1,2, \ldots, M$ Doppler frequency bands using the associated steering vector $\mathbf{s}_{m}$ as

$$
\operatorname{ACE}_{\mathrm{CTD}}\left(m, k_{c}\right)=\frac{\left|\mathbf{s}_{m}^{H} \tilde{\mathbf{R}}_{\mathrm{U}}^{-1} \mathbf{z}_{\mathrm{CTD}, k_{c}}\right|^{2}}{\left(\mathbf{s}_{m}^{H} \tilde{\mathbf{R}}_{\mathrm{U}}^{-1} \mathbf{s}_{m}\right)\left(\mathbf{z}_{\mathrm{CTD}, k_{c}}^{H} \tilde{\mathbf{R}}_{\mathrm{U}}^{-1} \mathbf{z}_{\mathrm{CTD}, k_{c}}\right)} .
$$

It is this value that is to be compared with the ACE threshold to determine if a target exists at a particular range cell and Doppler.

To determine the level of the ACE threshold we compute a quiescent ACE level which corresponds to the $H_{0}$ hypothesis where no target is present. This is accomplished by determining the ACE values for the block of $k_{u}=1,2, \ldots, K_{u}$ local uncensored snapshots $\mathbf{z}_{\mathrm{UTD}, k_{u}}$ for each of the $m=1,2, \ldots, M$ Doppler frequency bands as

$$
\operatorname{ACE}_{\mathrm{UTD}}\left(m, k_{u}\right)=\frac{\left|\mathbf{s}_{m}^{H} \tilde{\mathbf{R}}_{\mathrm{I}}^{-1} \mathbf{z}_{\mathrm{UTD}, k_{u}}\right|^{2}}{\left(\mathbf{s}_{m}^{H} \tilde{\mathbf{R}}_{\mathrm{I}}^{-1} \mathbf{s}_{m}\right)\left(\mathbf{z}_{\mathrm{UTD}, k_{u}}^{H} \tilde{\mathbf{R}}_{\mathrm{I}}^{-1} \mathbf{z}_{\mathrm{UTD}, k_{u}}\right)} .
$$

To set the local relative quiescent level for the $M$ Doppler bins of the ACE detector we average the 


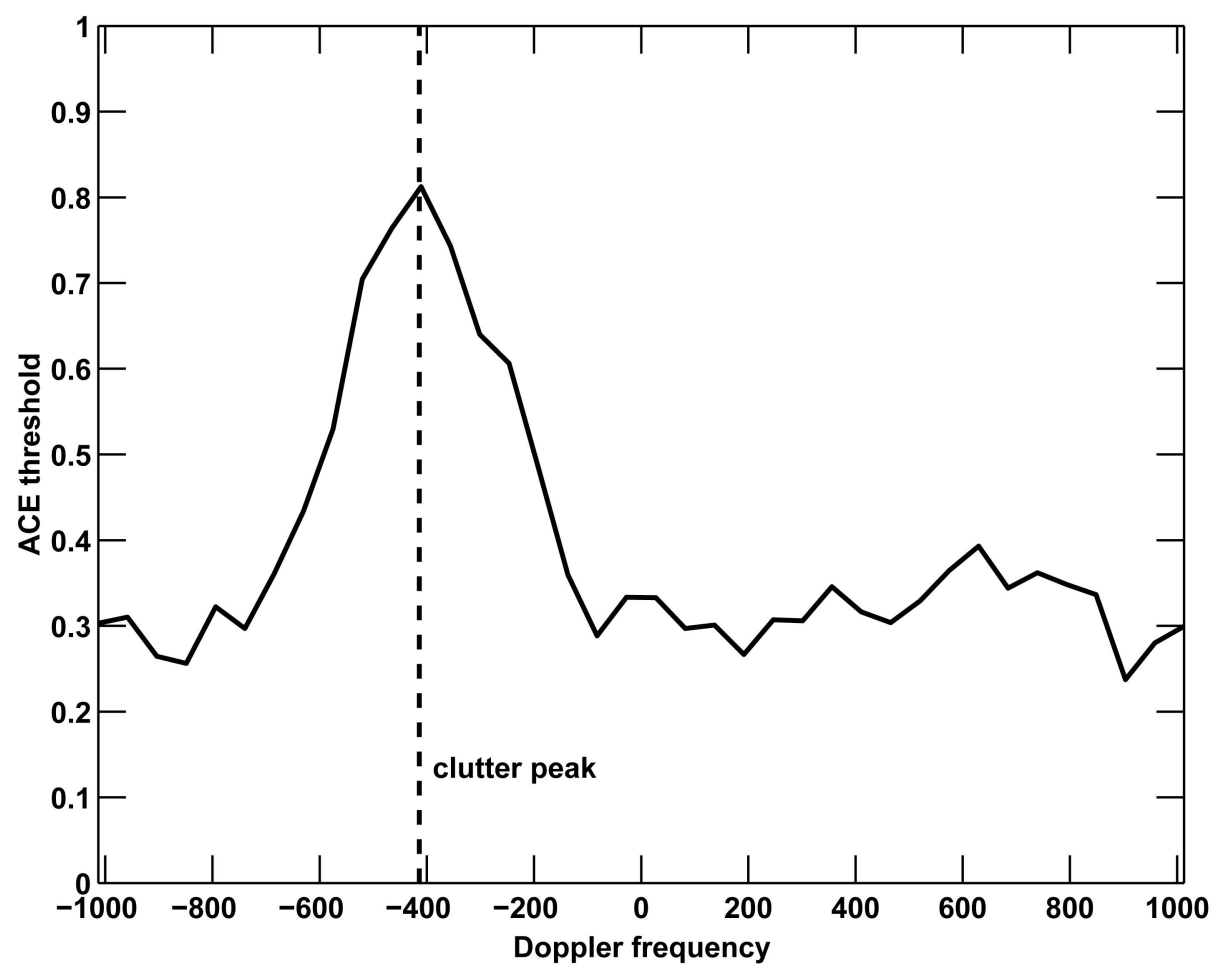

Fig. 2. DS-ACE computed threshold for KASSPER II.

local UTD ACE values for each Doppler over the block of local uncensored range cells which yields the $M$-length vector

$$
\gamma=\left[\begin{array}{c}
\frac{1}{K_{u}} \sum_{k_{u}=1}^{K_{u}} \operatorname{ACE}_{\mathrm{UTD}}\left(1, k_{u}\right) \\
\vdots \\
\frac{1}{K_{u}} \sum_{k_{u}=1}^{K_{u}} \operatorname{ACE}_{\mathrm{UTD}}\left(M, k_{u}\right)
\end{array}\right]
$$

in which each element of the vector will lie between 0 and 1. As an example, Fig. 2 illustrates the ACE threshold values computed for KASSPER II in which the peak of the clutter ridge in Doppler from (12) and (13) is located at $-414 \mathrm{~Hz}$. In order to obtain the desired level of false alarm, $\gamma$ is scaled to yield the DS-ACE threshold vector $\tau=\alpha \gamma$ where $\alpha$ is a constant scale factor (across the Doppler bins) used to control the false alarm probability and is set such that the smallest value of $\tau$ is the minimum desired threshold level $\tau_{\min }$. Note that to some degree a nonadaptive approach similar to the DS-ACE is already employed in most pulse-Doppler radars whereby returns in Doppler bins near the clutter ridge peak are simply excluded from detection. The benefit of DS-ACE therefore lies in the fact that it adaptively controls the ACE threshold at the "shoulders" of the clutter ridge outside of the excluded Doppler bands where targets are detectable yet undernulled clutter may still exist.

\section{SIMULATION RESULTS}

In this section, the performance of the FRACTA.E algorithm supplemented with KACE and DS-ACE is evaluated using the KASSPER I challenge datacube [9] and CPI 22 of the KASSPER II data [10] (for which there is ground truth to compare). Other researchers $[36,37]$ have presented promising results for the KASSPER I datacube. Both KASSPER I and II datasets contain dense groups of targets which are known to degrade performance for the standard STAP processors. The simulated radar in KASSPER II has the added difficulty of being operated at a higher frequency with approximately the same PRF as the KASSPER I scenario which gives rise to significant aliasing of the clutter for the KASSPER II dataset thereby resulting in a considerably higher concentration of clutter. Therefore, the clutter in KASSPER II requires more adaptive degrees-of-freedom to adequately suppress.

Due to range/Doppler sidelobes resulting from pulse compression and Doppler filtering, it is common for a target to spread into nearby range-Doppler cells. For this reason, it is standard procedure for a radar to cluster target detections such that a detection in a given range-Doppler cell is associated with a target that lies in a contiguous range cell or Doppler band. For the results reported here, we cluster \pm 1 cells in range and \pm 1 bands in Doppler for all processing schemes. In other words, if a censored range/Doppler cell which exceeds both the CFAR and ACE thresholds falls within the $3 \times 3$ range/Doppler 


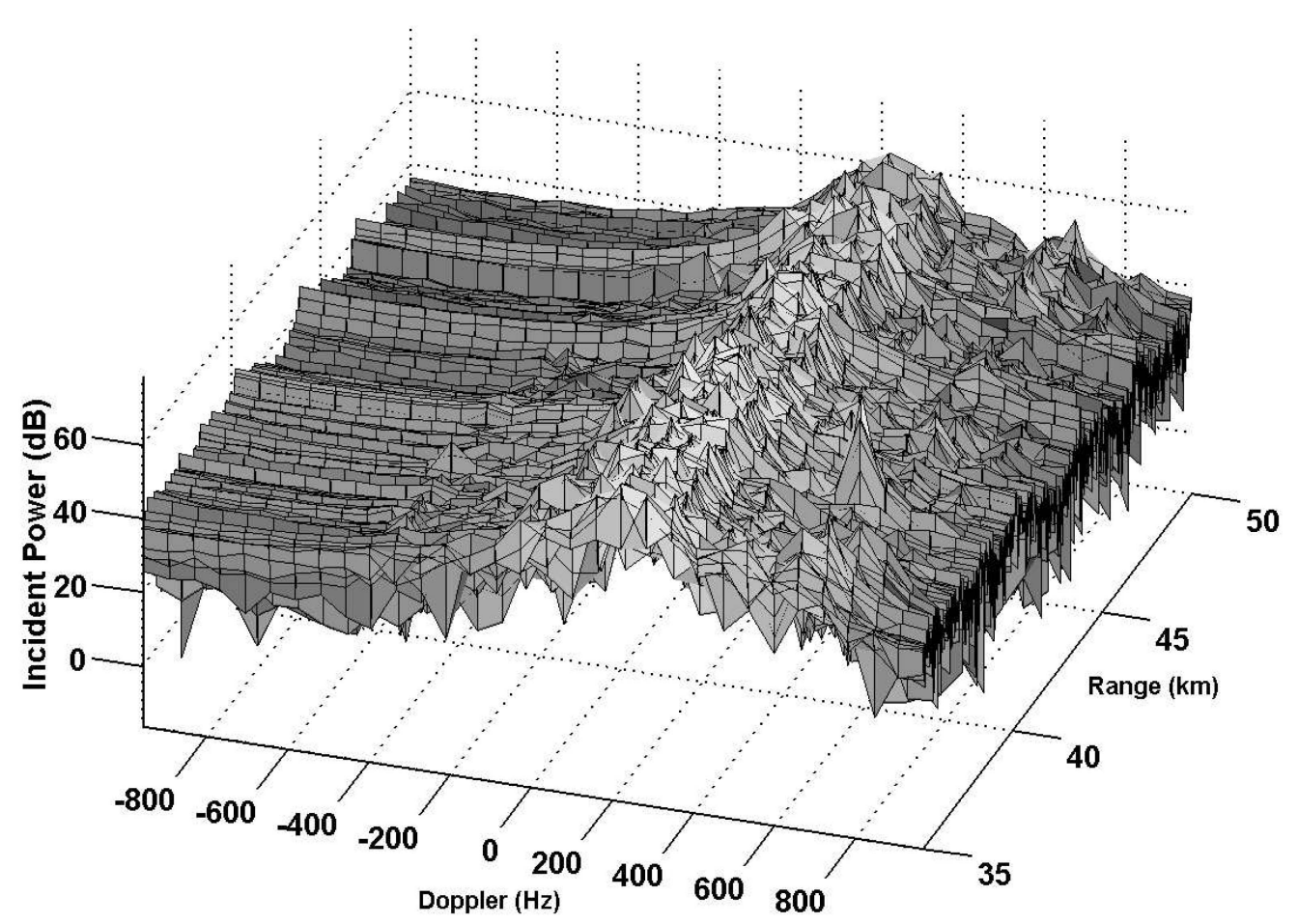

Fig. 3. Incident power versus range and Doppler for KASSPER I.

grid centered on a real target (based upon the available KASSPER ground truth) it is declared a true detection. If not, it is deemed a false alarm. Note that in this way several targets may be counted from a single detection but each is counted only once (i.e., no repetition of counted targets). While in practice these targets could not be separately counted, this approach provides an easy means for comparison in terms of target detection performance.

For both KASSPER I and II data sets, clairvoyant knowledge results are obtained by applying the available clairvoyant covariance matrices available with the respective data sets. The clairvoyant covariance matrices contain the true second-order statistical information of the clutter for each individual range cell. Following the application of the weight vector that results from using the clairvoyant covariance matrix, the ACE metric is computed and compared with a threshold to determine if it is to be declared a detection.

\section{A. Performance of FRACTA.E-KACE for KASSPER I}

The incident power on the first antenna element for KASSPER I is presented in Fig. 3. The operating parameters for KASSPER I are given in Table I. The KASSPER I dataset is simulated high-fidelity airborne radar data for the nonhomogeneous terrain near Olancha, CA which consists of mountains and deserts. The average power incident upon a single antenna element relative to the noise floor is depicted in Fig. 4 in which the dynamic range is found to be roughly $28 \mathrm{~dB}$ and the average power is $37 \mathrm{~dB}$ above the noise floor. The peaks and troughs in range are believed to be the result of shadowing due to mountains. The simulated airborne radar was flying at $3000 \mathrm{~m}$ altitude with an average velocity of $100 \mathrm{~m} / \mathrm{s}$ traveling due east $\left(270^{\circ}\right.$ measured from true north) with a $3^{\circ}$ average crab angle. Some random fluctuation was present in the platform velocity and crab angle representative of actual platform motion over the CPI. The 11 (virtual) antenna array elements were spaced slightly less than a half-wavelength apart at $0.1092 \mathrm{~m}$ (0.9028 half-wavelength spacing), and the antenna boresight was pointed at $177^{\circ}$ with a $5^{\circ}$ depression angle. The radar operating parameters can be found in Table I which were those employed for the KACE covariance matrix in (7) and are thus inclusive of any errors introduced due to platform motion and array calibration errors which are on the order of 5-10 deg. Finally, the KASSPER I dataset is found to possess a sample covariance matrix with roughly 50 dominant eigenvalues so that according to the $2 J$ rule established in [16], the required sample support is at least 100 samples.

It has been shown in [4] that given sufficient sample support for the KASSPER I datacube the FRACTA.E approach is capable of attaining nearly the same target detection performance as when clairvoyant knowledge of the clutter is available (which it is not in practice). Fig. 5 illustrates the effects of KACE on FRACTA.E in terms of target detections for a single false alarm as a function of sample support for the computation of the AMF weight vectors. FRACTA.E requires roughly 100 samples at which 


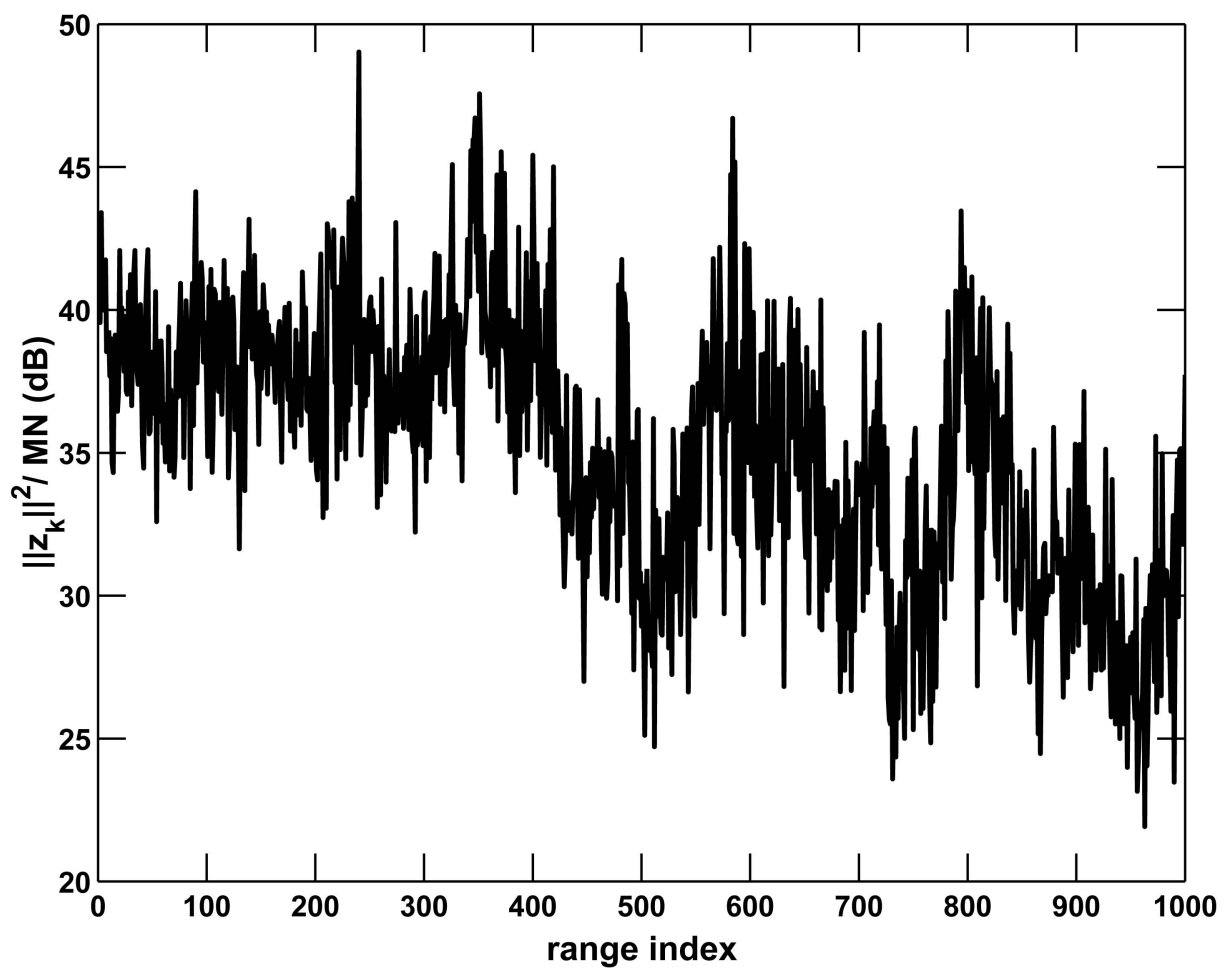

Fig. 4. Range profile of incident power for KASSPER I.

TABLE I

Operating Parameters for KASSPER I High-Fidelity Datacube

\begin{tabular}{lc}
\hline \multicolumn{1}{c}{ Parameter } & Value \\
\hline carrier frequency & $1240 \mathrm{MHz}$ \\
bandwidth & $10 \mathrm{MHz}$ \\
number of pulses $(M)$ & 32 \\
number of array channels $(N)$ & 11 \\
minimum range & $35 \mathrm{~km}$ \\
maximum range & $50 \mathrm{~km}$ \\
number of range cells & 1000 \\
pulse repetition frequency & $1984 \mathrm{~Hz}$ \\
beta & 0.923 \\
peak power & $15 \mathrm{~kW}$ \\
platform speed & $100 \mathrm{~m} / \mathrm{s}$ \\
crab angle & $3^{\circ}$ \\
\hline
\end{tabular}

point it detects 192 out of the 268 targets present for a single false alarm, the same as clairvoyant. However, when KACE is used to supplement the AMF covariance matrix the sample support can be as low as 30 and near-clairvoyant target detection performance is maintained. Also, FRACTA.E with and without KACE significantly outperforms the SWP method using LSMI primarily due to the density of targets which SWP does not censor from the training data.

When the DS-ACE detector is used, the detection performance of FRACTA.E with and without KACE for a single false alarm is shown in Fig. 6. While the FRACTA.E algorithm with DS-ACE does not quite reach clairvoyant performance (because the threshold is now estimated from the data), it does achieve a higher number of target detections for low sample support (steady state reached at $\sim 60$ samples) than when DS-ACE was not employed. Also, supplementing FRACTA.E with KACE results in detection performance that is close to clairvoyant for a sample support of only 20. Furthermore, with zero sample support (i.e., essentially nonadaptive) 144 targets are detected by FRACTA.E with KACE and DS-ACE whereas 100 are detected when DS-ACE is not used. As expected, KACE improves performance by replacing some of the deficient clutter covariance information when sample support is low. Also, the DS-ACE detector is found to add some additional robustness to the FRACTA.E algorithm since it reduces the probability of false alarms being detected near the clutter ridge where undernulled clutter may exist, especially for low sample support.

\section{B. Performance of FRACTA.E-KACE for KASSPER II}

The incident power on the first antenna element for KASSPER II is presented in Fig. 7. The KASSPER II datacube is also simulated highfidelity airborne radar data for the nonhomogeneous mountainous/desert terrain near Olancha, CA. The average power incident upon a single antenna element relative to the noise floor is depicted in Fig. 8 where the dynamic range is roughly $50 \mathrm{~dB}$ and the average clutter power is $29 \mathrm{~dB}$ above the noise floor. Compared with KASSPER I, the KASSPER II datacube suffers from more severe shadowing with deep nulls such as near range index 1400. The 


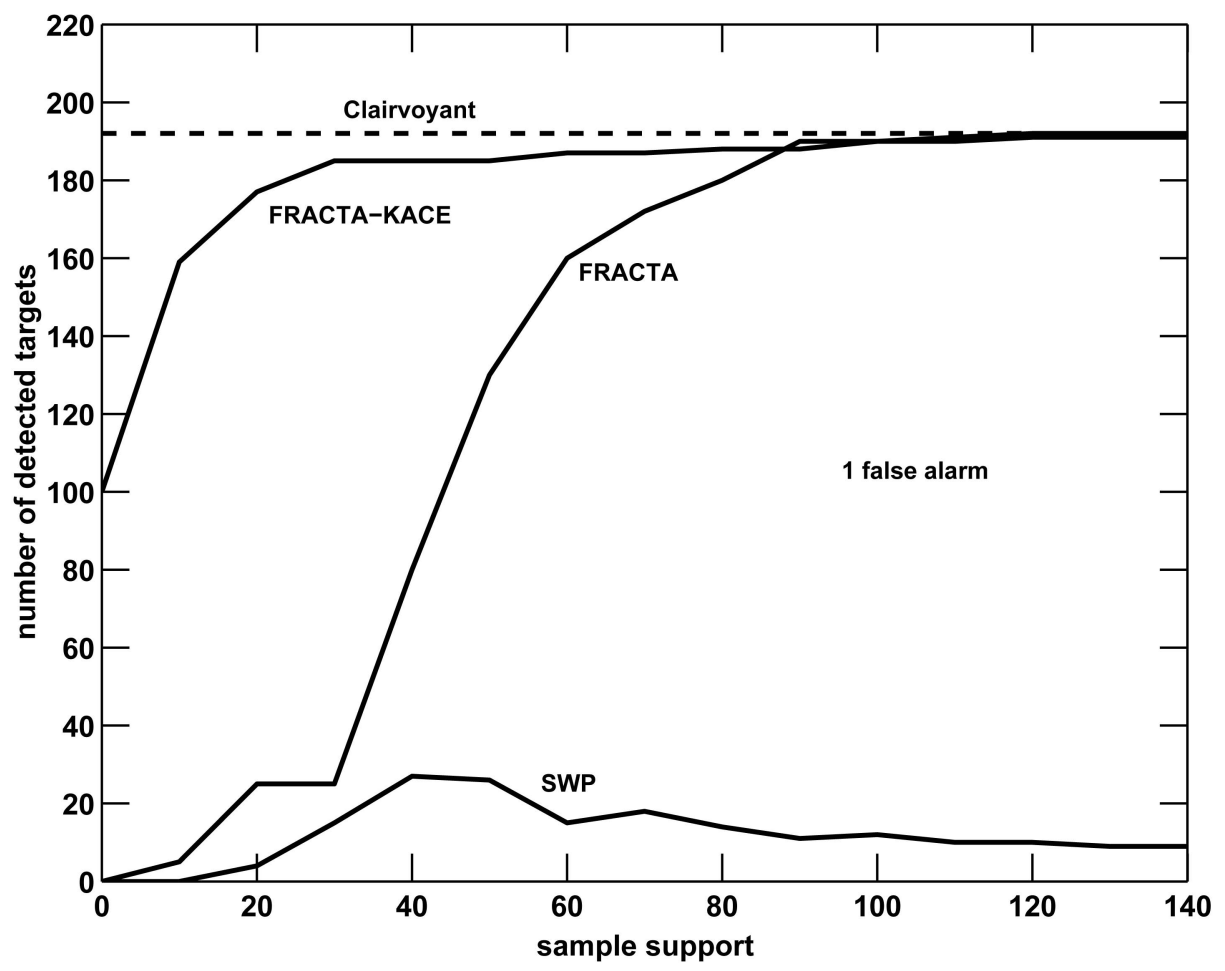

Fig. 5. FRACTA.E with/without KACE applied to KASSPER I.

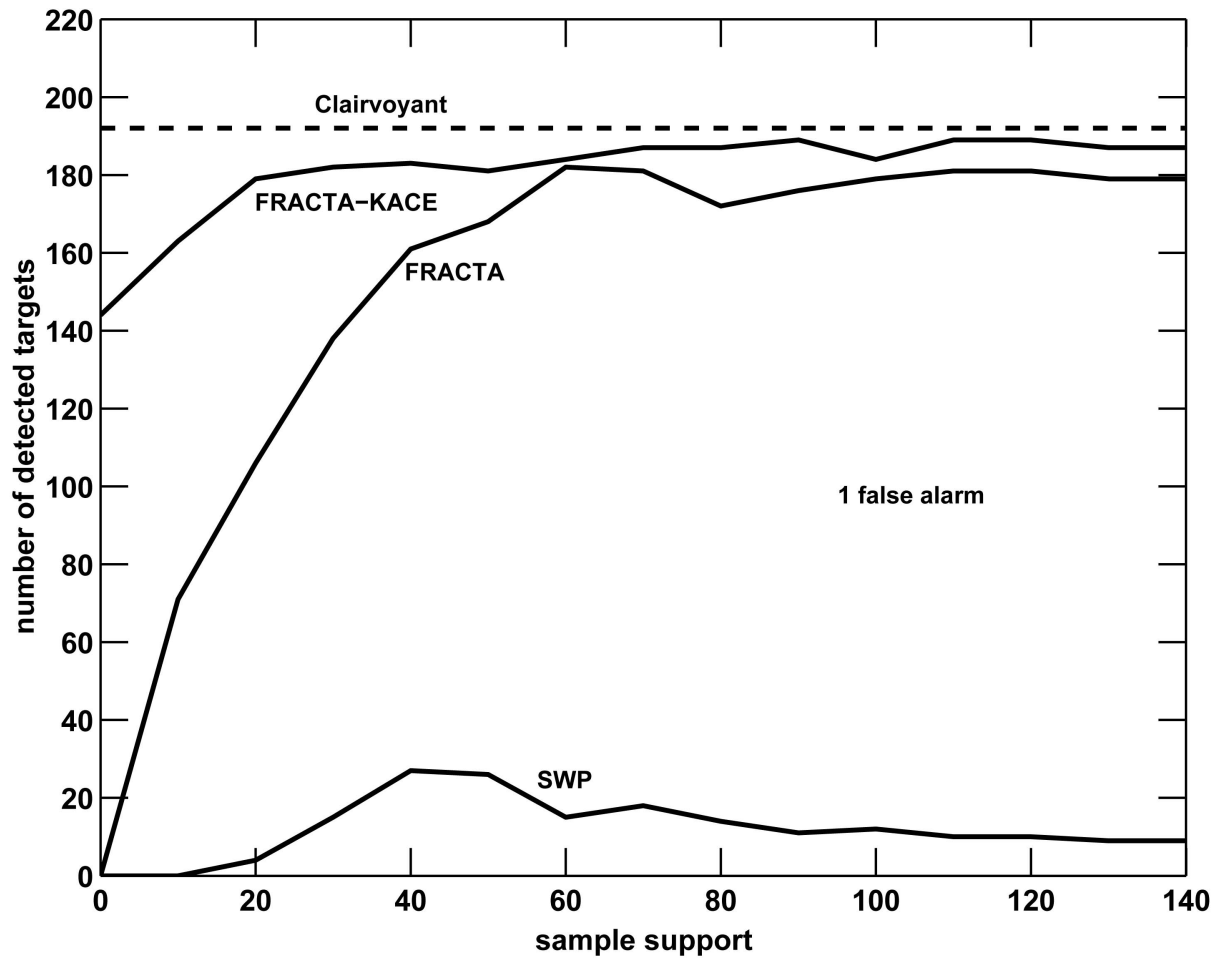

Fig. 6. FRACTA.E using DS-ACE with/without KACE applied to KASSPER I.

simulated airborne radar was flying at $7000 \mathrm{~m}$ altitude traveling at an average velocity of $150 \mathrm{~m} / \mathrm{s}$ due east $\left(270^{\circ}\right.$ measured from true north) with a $3^{\circ}$ average crab angle. The 12 input channels are obtained after subarray beamforming on 96 antenna array elements in which the antenna elements were spaced exactly a half-wavelength apart, and the antenna boresight was pointed at $182^{\circ}$ with a $5^{\circ}$ depression angle. The operating parameters for KASSPER II are given in Table II. The sample covariance matrix for KASSPER II is found to possess roughly 150 dominant eigenvalues. 


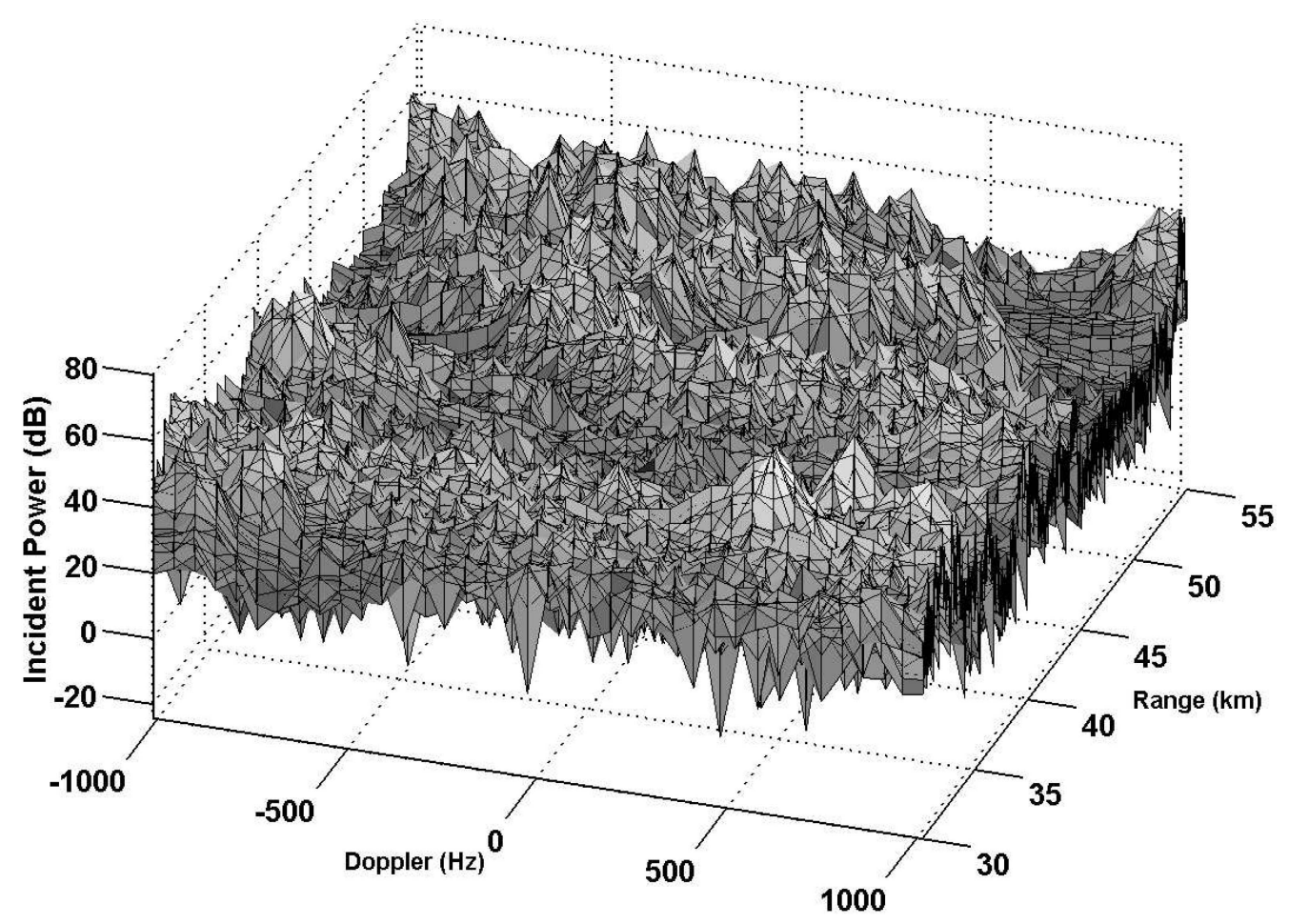

Fig. 7. Incident power versus range and Doppler for KASSPER II.

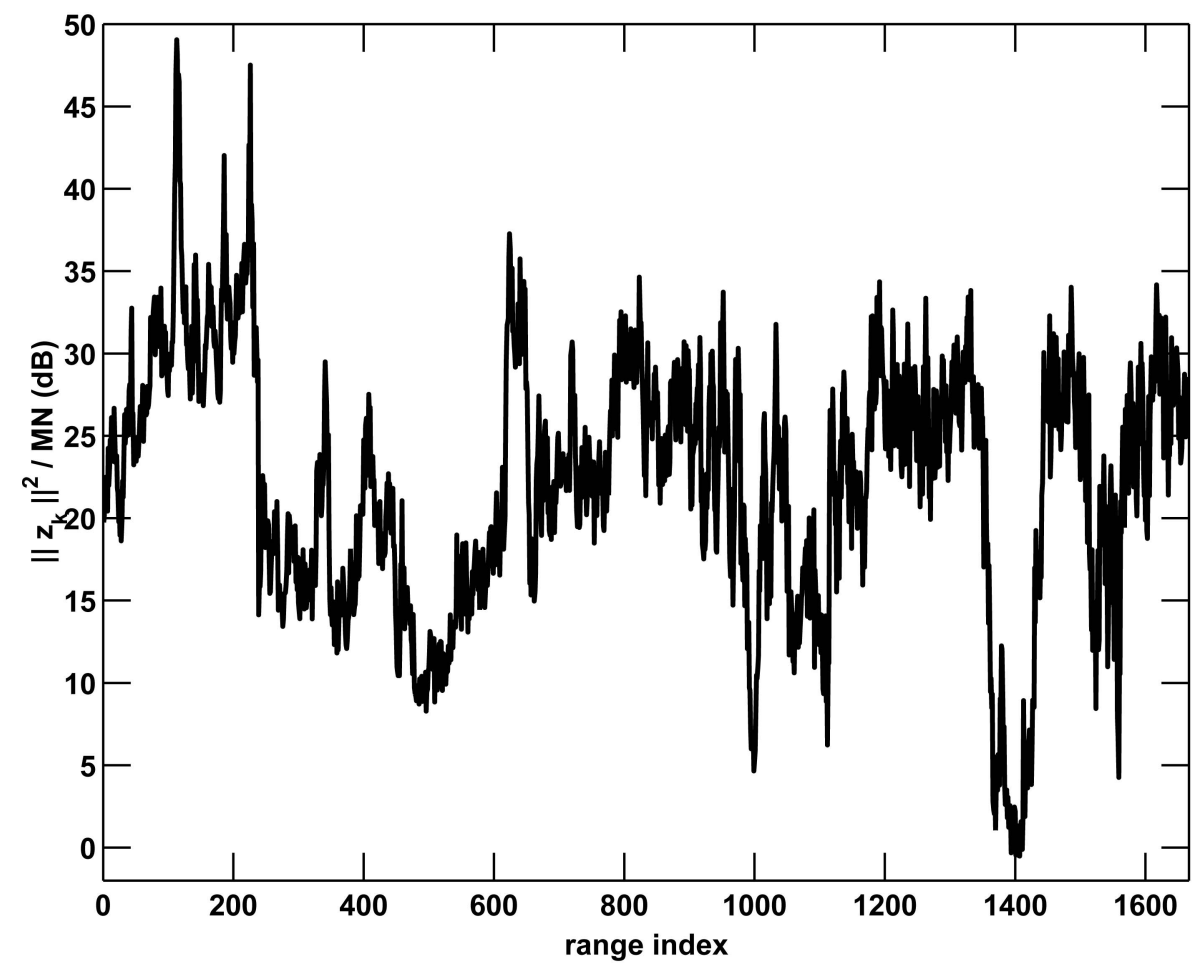

Fig. 8. Range profile of incident power for KASSPER II.

For KASSPER II we examine the performance of FRACTA.E using DS-ACE with and without KACE. For the KASSPER II dataset, the relatively low PRF for the $10 \mathrm{GHz}$ operating frequency causes Doppler aliasing of the clutter. The result is a less distinct clutter ridge in range/Doppler space. Therefore, it is likely that substantial undernulled clutter will exist after the application of the AMF weight vector. It is common practice for pulse-Doppler radar to censor a number of the Dopplers surrounding the expected location of the clutter ridge peak (which can be estimated via (12) and (13) for a given space-time steering vector) as the false alarms generated in those Dopplers would greatly outweigh the possibility 


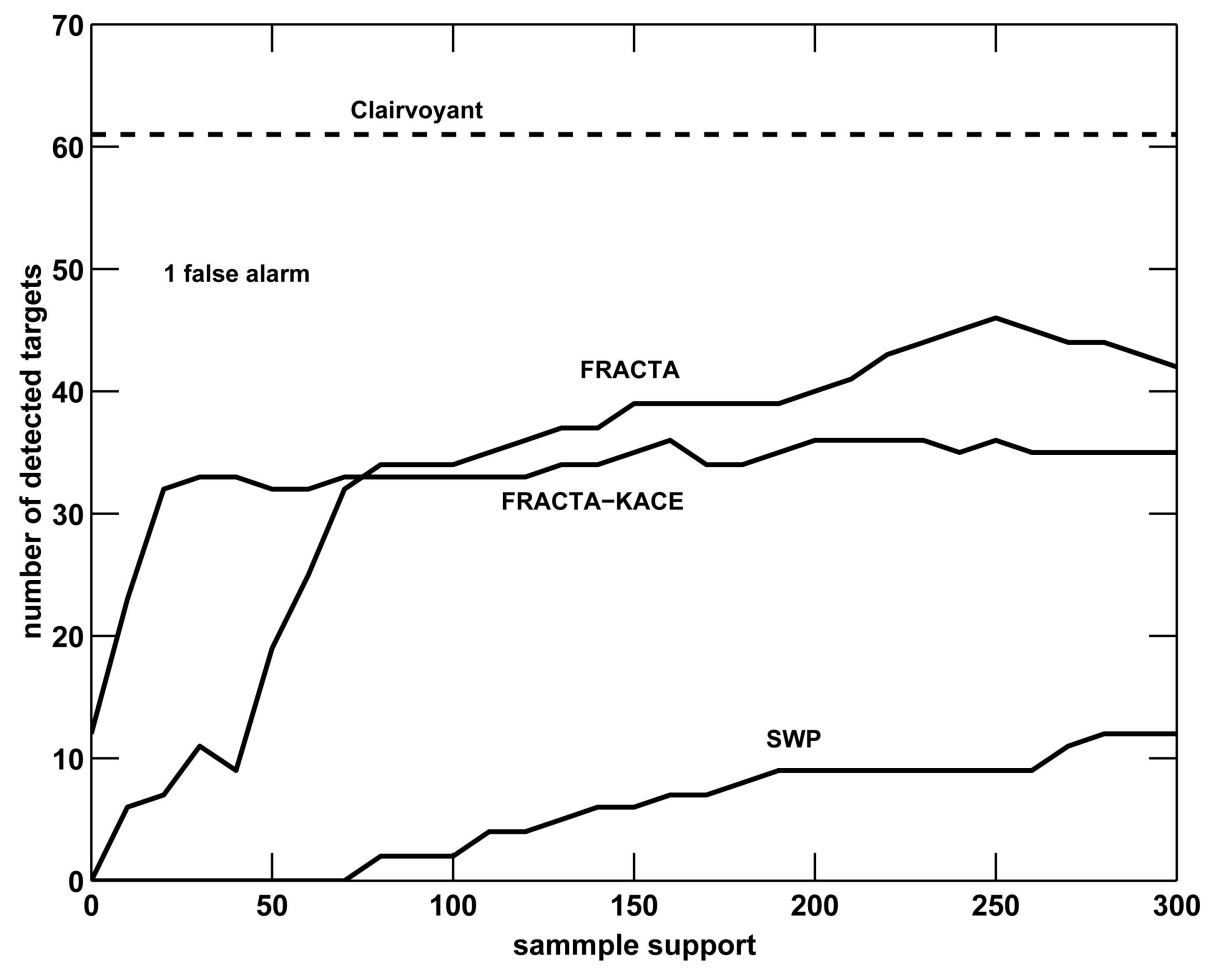

Fig. 9. FRACTA.E with/without KACE applied to KASSPER II.

TABLE II

Operating Parameters for KASSPER II High-Fidelity Datacube

\begin{tabular}{lc}
\hline \multicolumn{1}{c}{ Parameter } & Value \\
\hline carrier frequency & $10 \mathrm{GHz}$ \\
bandwidth & $10 \mathrm{MHz}$ \\
number of pulses $(M)$ & 38 \\
number of array channels $(N)$ & 12 \\
minimum range & $30 \mathrm{~km}$ \\
maximum range & $55 \mathrm{~km}$ \\
number of range cells & 1667 \\
pulse repetition frequency & $2081 \mathrm{~Hz}$ \\
beta & 9.616 \\
peak power & $10 \mathrm{~kW}$ \\
platform speed & $150 \mathrm{~m} / \mathrm{s}$ \\
crab angle & $3^{\circ}$ \\
\hline
\end{tabular}

of detecting targets. To that end, the returns in the Doppler bin corresponding to the peak of the clutter ridge and 3 Doppler bins on either side of the peak are not considered for detection. The DS-ACE detector therefore mitigates the undernulled clutter around the "shoulders" of the clutter ridge.

Using clairvoyant knowledge of the clutter covariance matrices yields 82 detected targets out of the 127 targets present for a single false alarm. However, 21 of these targets lie within a 3 range cell cluster almost exactly at the peak of the clutter ridge. Therefore, eliminating the 21 targets at the clutter ridge peak since they are apparently not moving, the clairvoyant case yields 61 target detections for a single false alarm. As depicted in Fig. 9, the FRACTA.E algorithm detects 47 targets for a single false alarm when the sample support reaches 250 . When KACE is employed to supplement FRACTA.E, the number of target detections drops to 36 for the same sample support. However, KACE provides as many as 25 more target detections when the sample support is low (less than 50).

As illustrated in Fig. 10, using DS-ACE with FRACTA.E boosts the number of detection for a single false alarm to 55 when the sample support reaches 250. When using KACE as well, the same level of detection performance is achieved for a sample support of 80, a 3-fold reduction in required sample support. Hence, even for severely aliased clutter, the FRACTA.E algorithm coupled with KACE and DS-ACE achieves near-clairvoyant performance (outside of the clutter peak) even for very low sample support.

\section{CONCLUSIONS}

The FRACTA.E has been shown previously to be an effective STAP methodology for the airborne/space-based radar configuration. In this paper, the performance of FRACTA.E was examined when low sample support is required due to heterogeneity of the interference. To enable satisfactory performance at low sample support, FRACTA.E was supplemented with prior knowledge via KACE which supplies some of the clutter covariance information that is deficient due to low sample support. In addition to KACE, a DS-ACE detector was introduced that provides 


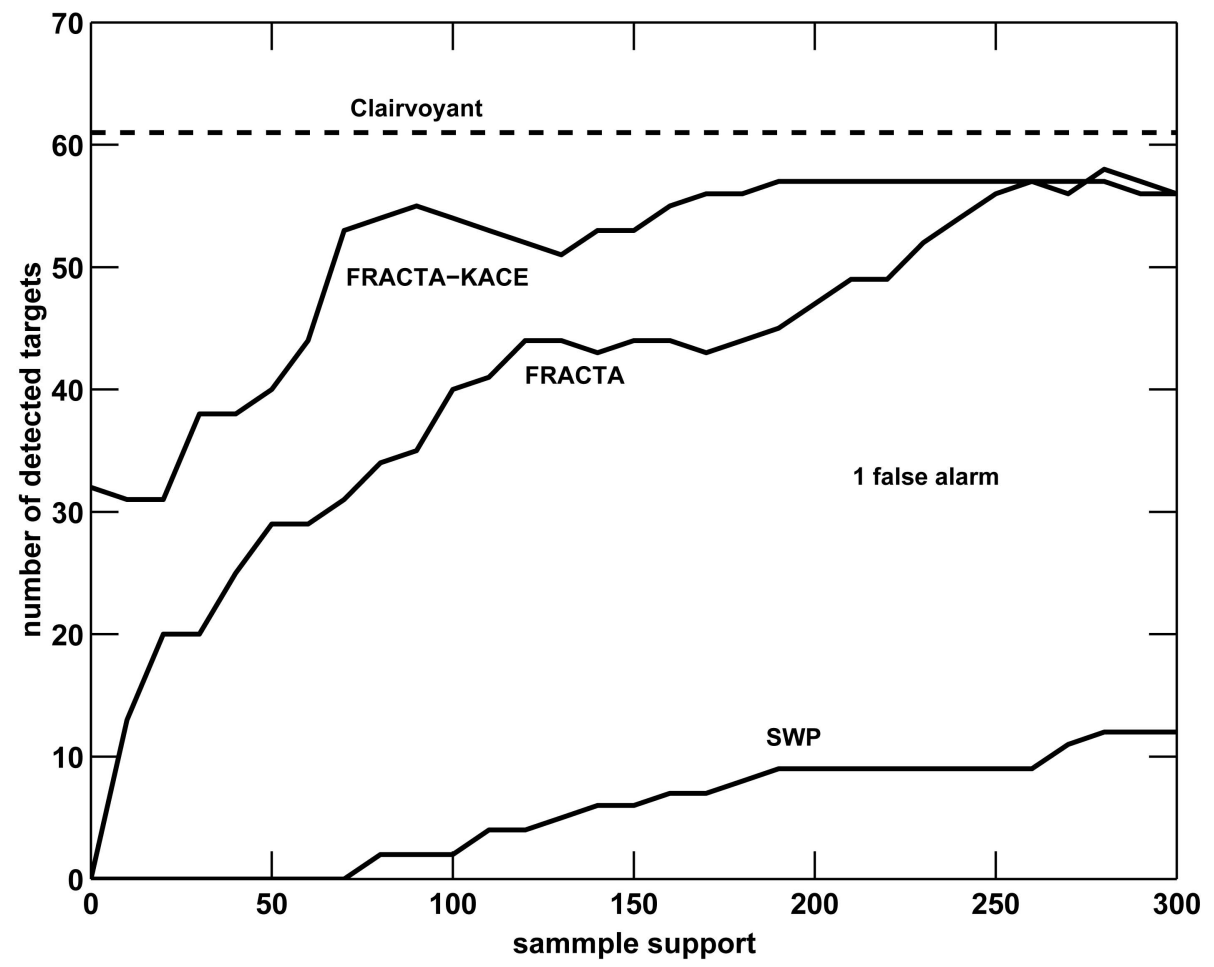

Fig. 10. FRACTA.E using DS-ACE with/without KACE applied to KASSPER II.

further robustness for the FRACTA.E algorithm to undernulled clutter which is especially problematic at low sample support. For the KASSPER I \& II high-fidelity simulated radar datasets, the combination of KACE and DS-ACE allowed the FRACTA.E algorithm to achieve near-clairvoyant performance for sample support as low as $1 / 3$ of that normally required to achieve near ideal detection performance.

\section{REFERENCES}

[1] Gerlach, K., and Picciolo, M. L.

Robust STAP using RC.

In Proceedings of IEEE National Radar Conference,

Huntsville, AL, May 5-8, 2003, 244-251.

[2] Gerlach, K., Blunt, S. D., and Picciolo, M. L.

Robust adaptive matched filtering using the FRACTA algorithm.

IEEE Transactions on Aerospace and Electronic Systems, 30, 3 (July 2004), 929-945.

[3] Blunt, S. D., and Gerlach, K.

Efficient RC of robust STAP using the FRACTA algorithm.

In Proceedings of IEEE International Conference on Radar, Adelaide, Australia, Sept. 3-5, 2003, 57-61.

[4] Blunt, S. D., and Gerlach, K.

Efficient robust adaptive matched filtering using the FRACTA algorithm: Results from KASSPER I.

To be published in IEEE Transactions on Aerospace and Electronic Systems.

[5] Gerlach, K., and Picciolo, M. L.

SINR improvement in airborne/spaceborne STAP using a priori platform knowledge.

In Proceedings of IEEE SAM Conference, Aug. 2002, 548-552.
[6] Gerlach, K., and Picciolo, M. L.

Airborne/spacebased radar STAP using a structured covariance matrix.

IEEE Transactions on Aerospace and Electronic Systems, 39, 1 (Jan. 2003), 269-281.

[7] Blunt, S. D., Gerlach, K., and Rangaswamy, M. The enhanced FRACTA algorithm with knowledge-aided covariance estimation.

Presented at the IEEE Sensor Array \& Multichannel Signal Processing Workshop, July 2004.

[8] Blunt, S. D., and Gerlach, K.

Efficient robust AMF using the enhanced FRACTA algorithm: Results from KASSPER I \& II. In Proceedings of IEEE Radar Conference, Philadelphia, PA, Apr. 26-29, 2004, 372-377.

[9] Bergin, J. S., and Techau, P. M.

High-fidelity site-specific radar simulation:

KASSPER '02 workshop datacube.

Technical note ISL-SCRD-TR-02-105, ISL, Vienna, VA, May 2002.

[10] Bergin, J. S., Techau, P. M., and Chaney, G.

High-fidelity site-specific radar simulation:

KASSPER data set 2.

ISL Technical note ISL-SCRD-TR-02-106, Vienna, VA, Oct. 2002.

[11] Maher, J., Callahan, M., and Lynch, D.

Effects of clutter modeling in evaluating STAP processing for space-based radars.

In Proceedings of IEEE International Radar Conference, 2000, 565-570.

[12] Babu, B. N. S., Torres, J. A., and Melvin, W. L. Processing and evaluation of multichannel airborne radar measurements (MCARM) measured data. In Proceedings of IEEE International Symposium on Phased Array Systems and Technology, Oct. 1996, 395-399. 
[13] Brennan, L. E., and Reed, I. S.

Theory of adaptive radar.

IEEE Transactions on Aerospace and Electronic Systems,

AES-9, 2 (Mar. 1973), 237-252.

[14] Reed, I. S., Mallet, J., and Brennan, L.

Rapid convergence rate in adaptive arrays.

IEEE Transactions on Aerospace and Electronic Systems,

AES-10 (Nov. 1974), 853-863.

[15] Carlson, B. D.

Covariance matrix estimation errors and diagonal loading in adaptive arrays.

IEEE Transactions on Aerospace and Electronic Systems, 24, 4 (July 1988), 397-401.

[16] Kirsteins, I. P., and Tufts, D. W.

Adaptive detection using low rank approximations to a data matrix.

IEEE Transactions on Aerospace and Electronic Systems, 30, 1 (Jan. 1994), 55-67.

[17] Haimovich, A.

The eigencanceler: Adaptive radar by eigenanalysis methods.

IEEE Transactions on Aerospace and Electronic Systems, 32, 2 (Apr. 1996), 532-542.

[18] Goldstein, J. S., Reed, I. S., and Scharf, L. L.

A multistage representation of the Wiener filter based on orthogonal projections.

IEEE Transactions on Information Theory, 44, 7 (Nov. 1998), 2943-2959.

[19] Guerci, J. R., Goldstein, J. S., and Reed, I. S. Optimal and adaptive reduced-rank STAP. IEEE Transactions on Aerospace and Electronic Systems, 36, 2 (Apr. 2000), 647-663.

[20] Steiner, M. J., and Gerlach, K.

Fast converging adaptive canceller for a structured covariance matrix.

IEEE Transactions on Aerospace and Electronic Systems, 36, 4 (Oct. 2000), 1115-1126.

[21] DiPietro, R. C.

Extended factored space-time processing for airborne radar systems.

In Record of the Twenty-Sixth Asilomar Conference on Signals, Systems, and Computers, vol. 1, Oct. 26-28, 1992, 425-430.

[22] Adve, R. S., Hale, T. B., and Wicks, M. C.

Practical joint domain localised adaptive processing in homogeneous and nonhomogeneous environments. 2. Nonhomogeneous environments.

IEE Proceedings, Pt. F, 147, 2 (Apr. 2000), 66-74.

[23] Ward, J.

Space-time adaptive processing for airborne radar.

Technical report ESC-TR-94-109, MIT Lincoln Lab, Dec. 1994.

[24] Kraut, S., Scharf, L. L., and McWhorter, L. T.

Adaptive subspace detectors.

IEEE Transactions on Signal Proceedings, 49, 1 (Jan. 2001), 1-16.

[25] Melvin, W. L., Showman, G. A., and Guerci, J. R.

A knowledge-aided GMTI detection architecture.

In Proceedings of IEEE Radar Conference, Apr. 26-29,

2004, 301-306.
[26] Bergin, J. S., Teixeira, C. M., Techau, P. M., and Guerci, J. R.

STAP with knowledge-aided data prewhitening.

In Proceedings of IEEE Radar Conference, Apr. 26-29, 2004, 289-294.

[27] Letgers, G. R., and Guerci, J. R.

Physics-based airborne GMTI radar signal processing.

In Proceedings of IEEE Radar Conference, Apr. 26-29, 2004, 283-288.

[28] Bergin, J. S., Techau, P. M., Melvin, W. L., and Guerci, J. R. GMTI STAP in target-rich environments: Site-specific analysis.

In Proceedings of IEEE Radar Conference, Apr. 22-25, 2002, 391-396.

[29] Antonik, P., Schuman, H., Li, P., Melvin, W. L., and

Wicks, M.

Knowledge-based space-time adaptive processing.

In Proceedings of IEEE Radar Conference, May 13-15, 1997, 372-377.

[30] Goodman, N. A., and Gurram, P. R.

STAP training through knowledge-aided predictive modeling.

In Proceedings of IEEE Radar Conference, Apr. 26-29, 2004, 388-393.

[31] Moon, T. K., and Stirling, W. C.

Mathematical Methods and Algorithms for Signal

Processing,

Upper Saddle River, NJ: Prentice-Hall, 2000, 258-261.

[32] Blunt, S. D., and Gerlach, K.

Robust adaptive matched filtering using the

FRACTA algorithm: Results from RLSTAP and

KASSPER high-fidelity clutter models.

Technical report 10,059, U.S. Naval Research Laboratory, Washington, D.C.

[33] Barton, D. K. (Ed.)

Radar Clutter (Radars, vol. 5).

Norwood, MA: Artech House, 1975.

[34] Billingsley, J. B.

Low-Angle Radar Land Clutter: Measurements and Empirical Models.

Norwich, NY: William Andrew Publishing, 2002.

[35] Hiemstra, J. D.

Colored diagonal loading.

In Proceedings of IEEE National Radar Conference, Apr. 22-25, 2002, 386-390.

[36] Teixeira, C. M., Bergin, J. S., and Techau, P. M.

Adaptive thresholding of non-homogeneity detection for STAP applications.

In Proceedings of IEEE National Radar Conference, Apr. 26-29, 2004, 355-360.

[37] Li, P., Schuman, H., Michels, J. H., and Himed, B. Space-time adaptive processing (STAP) with limited sample support.

In Proceedings of IEEE National Radar Conference, Apr. 26-29, 2004, 366-371. 

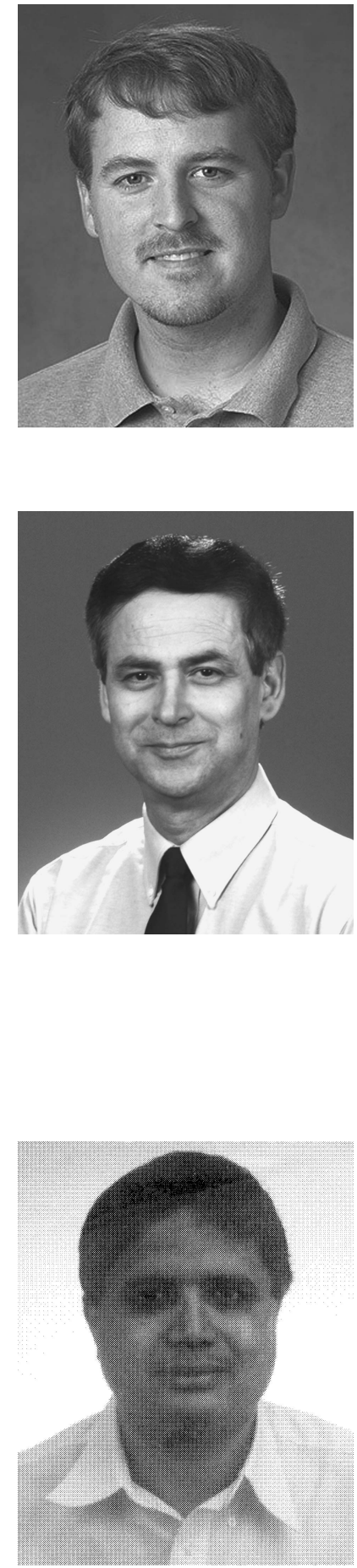

Shannon D. Blunt (S'96-M'02) received the B.S., M.S., and Ph.D. degrees in electrical engineering in 1999, 2000, and 2002, respectively, from the University of Missouri-Columbia (MU).

From 2002 to 2005 he was with the Radar Division of the U.S. Naval Research Laboratory in Washington, D.C. In 2005 he joined the faculty of the Department of Electrical Engineering and Computer Science at the University of Kansas.

Dr. Blunt received the Donald K. Anderson Graduate Student Teaching award in electrical engineering from MU in 2000 and the MU Outstanding Graduate Student award in electrical engineering in 2001. He also received the 2004 Naval Research Laboratory Alan Berman Research Publication award. He is a member of Eta Kappa Nu and Tau Beta Pi. His research interests are in adaptive signal processing for radar and communications with an emphasis on waveform diversity techniques.

Karl Gerlach (M'81-F'02) was born in Chicago, IL. He received his B.S. in 1972 from the University of Illinois, Urbana, and his M.S. and D.Sc. from George Washington University, Washington, D.C., in 1975 and 1981, respectively, all in electrical engineering.

Since 1972, he has been employed by the Naval Research Laboratory in Washington, D.C. From 1972 to 1976, he worked on experimental submarine communications systems and from 1976 to the present he has been with the Radar Division where his research interests include adaptive signal processing and space-based radar.

Dr. Gerlach was the 1986 recipient of the IEEE AESS Radar Systems Panel award.

Muralidhar Rangaswamy (S'89-M'90-SM'98—F'05) received the Ph.D. degree in electrical engineering from Syracuse University, Syracuse, NY, in 1992.

$\mathrm{He}$ is currently employed as a senior electronics engineer at the Air Force Research Laboratory (AFRL), Hanscom AFB, MA, where he is conducting research in the area of phenomenology-based adaptive radar signal processing. Prior to this, he held positions in the industry and academia.

Dr. Rangaswamy has authored more than forty journal and conference papers in the areas of his research. He has delivered invited presentations at numerous government, industrial and academic forums. He is a coinventor on 2 U.S. patents and is a coauthor of two book chapters. He is a member of the editorial board of the Digital Signal Processing journal and is an associate member of the SAM technical committee of the IEEE Signal Processing Society. He has organized numerous conferences, workshops, and short courses in the areas of his research. He received the postdoctoral research associate award from the National Research Council, USA in 1993. At AFRL, he has received 14 scientific achievement awards. 